\title{
DERECHO Y POLÍTICAS AMBIENTALES EN LA COMUNIDAD DE MADRID
}

(PRIMER SEMESTRE 2019)

\author{
DANIEL B. ENTRENA RUIZ \\ Profesor Contratado Doctor \\ Universidad Carlos III de Madrid
}


SUMARIO: 1. Estrategia de Gestión Sostenible de los Residuos de la Comunidad de Madrid 20172024. 2. Decreto 5/2019, de 5 de febrero, del Consejo de Gobierno, por el que se establece la acreditación profesional de los agentes forestales de la comunidad de Madrid. 3. Resolución de 5 de febrero de 2019, del Director General de Medio Ambiente y Sostenibilidad, de la Consejería de Medio Ambiente y Ordenación del Territorio, por la que se regula el tránsito por viales, el tránsito en las inmediaciones y pie de vía de sectores de escalada y la escalada en los sectores y vías regulados, en el paraje de La Pedriza del Parque Nacional de la Sierra de Guadarrama como medida de protección de especies rupícolas durante su época de cría y reproducción. 4. Resolución de 4 de febrero de 2019, de la Dirección General de Medio ambiente y Sostenibilidad, por la que se determinan las líneas eléctricas aéreas de alta tensión existentes en zonas de protección aprobadas en la Comunidad de Madrid, que no se ajustan a las prescripciones técnicas establecidas en el Real Decreto 1432/2008, de 29 de agosto, por el que se establecen medidas para la protección de la avifauna contra la colisión y la electrocución en líneas eléctricas de alta tensión 5. Decreto por el que se adoptan en la ciudad de Madrid las medidas de restricción del tráfico y del estacionamiento previstas en el protocolo de actuación para episodios de contaminación por dióxido de nitrógeno en la ciudad de Madrid.

\section{ESTRATEGIA DE GESTIÓN SOSTENIBLE DE LOS RESIDUOS DE LA COMUNIDAD DE MADRID 2017-2024}

Tras el agotamiento de la Estrategia Regional de Residuos 2002-2016 de la Comunidad de Madrid, en el BOCM del jueves 17 de enero de 2019, fue publicada la Resolución de 4 de enero de 2019, del Director General de Medio Ambiente y Sostenibilidad, mediante la que se daba publicidad a la aprobación de la Estrategia de Gestión Sostenible de los Residuos de la Comunidad de Madrid 2017-2024; de este modo se daba cumplimiento a lo establecido en el apartado segundo del artículo 26 de la Ley 21/2013, de 9 de diciembre, de Evaluación Ambiental, que preceptúa la publicación de todo plan o programa que posea efectos significativos sobre el medio ambiente.

La Estrategia aprobada orienta la política regional en materia de residuos, estableciendo las medidas necesarias para cumplir con los objetivos fijados en la normativa europea y española, el Plan Estatal Marco de Gestión de Residuos (PEMAR) 2016-2022 y el Programa Estatal de Prevención de Residuos (20142020), cuyo objetivo general es conseguir en 2020 una reducción del $10 \%$ en peso de los residuos generados respecto a los generados en el año 2010.

Conforme a este marco estable, la Estrategia aprobada presenta varios objetivos a general: Prevenir la generación de residuos en la Comunidad de Madrid; maximizar la transformación de los residuos en recursos, en aplicación de los 
principios de la economía circular, reducir el impacto ambiental asociado con carácter general a la gestión de los residuos y, en particular, los impactos vinculados al calentamiento global; fomentar la utilización de las Mejores Técnicas Disponibles en el tratamiento de los residuos; definir criterios para el establecimiento de las infraestructuras necesarias y para la correcta gestión de los residuos de la Comunidad de Madrid.

Tras establecer un Programa de Prevención de Residuos (2017-2024), la Estrategia contempla para este periodo un Plan Regional para cada una de las tipologías de residuos considerados, a saber: Domésticos y Comerciales, Industriales, de Construcción y Demolición, de Aparatos Eléctricos y Electrónicos, de Pilas y Acumuladores, Vehículos al Final de su Vida Útil, de Neumáticos Fuera de Uso, de Residuos de PCB, de Lodos de Depuración de Aguas Residuales, y de Suelos Contaminados

Dichos planes deberán a su vez ser desarrollados y ejecutados por los 179 municipios de la Comunidad de Madrid respecto aquellos residuos que sean de su competencia, bien individualmente bien asociadamente a través de las mancomunidades, contribuyendo así -todas las instituciones implicadas- a la disminución de los gases de efectos invernadero y en última instancia de la propia huella de carbono.

No obstante, no se han incluido en la Estrategia os residuos agrarios y los residuos de actividades extractivas; en el primer caso por representar un muy pequeño porcentaje en la Comunidad de Madrid, aunque según reconoce el propio documento que la contiene próximamente comenzarán los trabajos para diagnosticar su gestión de estos residuos y aprobar un plan específico. Por lo que respecta a las actividades extractivas, al quedar fuera de la Ley de Residuos y Suelos Contaminados (artículo 2.2), se contempla igualmente la elaboración y aprobación de un plan propio de gestión. 


\section{DECRETO 5/2019, DE 5 DE FEBRERO, DEL CONSEJO DE GOBIERNO, POR EL QUE SE ESTABLECE LA ACREDITACIÓN PROFESIONAL DE LOS AGENTES FORESTALES DE LA COMUNIDAD DE MADRID}

Mediante este Decreto se desarrolla la Ley 1/2002 de 27 de marzo, por la que se crea el cuerpo de agentes forestales de la comunidad de Madrid y se deroga la Orden 3364/2005, de 24 de noviembre, del sobre acreditación de los funcionarios del cuerpo de agentes forestales.

La Orden actualiza el modelo del documento de acreditación profesional de los agentes forestales de la Comunidad de Madrid, lo que incluye su placa identificativa en el uniforme, su documento de identificación en cuanto agente de la autoridad formalmente reconocido como tal, y la posibilidad de incorporar a la uniformidad otros elementos identificativos de organismos o ámbitos en los que los miembros del Cuerpo de Agentes Forestales prestan servicio.

La Orden no regula así la cualificación exigida a estos Agentes, pues viene determinada por la propia Ley de creación:

- A) Escala Técnica: que comprende las categorías de Técnico superior agente forestal (Grupo A) (Doctor, Licenciado, Ingeniero, Arquitecto o equivalente, con especialización en materias medioambientales o de protección y conservación de la naturaleza y Técnico medio agente forestal); y Técnico Medio agente Forestal (Grupo B) (Ingeniero Técnico, Diplomado Universitario, Arquitecto Técnico, Formación Profesional de tercer grado o equivalente, con especialización en materias medioambientales o de protección y conservación de la naturaleza)

- B) Escala Operativa, que comprende la Categoría de Agente Forestal, clasificada en el Grupo C: (título de Bachiller, Formación Profesional de segundo grado o equivalente)

3. RESOLUCIÓN DE 5 DE FEBRERO DE 2019, DEL DIRECTOR GENERAL DE MEDIO AMBIENTE Y SOSTENIBILIDAD, DE LA CONSEJERÍA DE MEDIO AMBIENTE Y ORDENACIÓN DEL TERRITORIO, POR LA QUE SE REGULA EL 
TRÁNSITO POR VIALES, EL TRÁNSITO EN LAS INMEDIACIONES Y PIE DE VÍA DE SECTORES DE ESCALADA Y LA ESCALADA EN LOS SECTORES $Y$ VÍAS REgULADOS, EN EL PARAJE DE LA PEDRIZA DEL PARQUE NACIONAL DE LA SIERRA DE GUADARRAMA COMO MEDIDA DE PROTECCIÓN DE ESPECIES RUPÍCOLAS DURANTE SU ÉPOCA DE CRÍA Y REPRODUCCIÓN

La regulación está motivada por la masificación de personas que acuden a las indicadas zonas de la Pedriza en las que existen nidos de buitre leonado (Gyps fulvus) y halcón peregrino (Falco peregrinus). Mediante esta resolución se aspira a proteger a estas aves durante el periodo de cría principalmente de molestias que pueden ocasionar los escaladores al utilizar itinerarios y riscos en los que las aves han localizado su nido.

En la resolución se describen las zonas en las que se restringe temporalmente la escalada por la presencia de nidos. Estas zonas son de dos tipos; en unas se res restringe la escalada por vías concretas (B1), mientras que en otras en las que existen especies más sensibles, se establece una restricción temporal en todo el sector (B2). De forma adicional también se regula el tránsito en ciertos viales que, por su proximidad a nidos, pueden ocasionar molestias (B3).

No obstante, la resolución contempla que fuera de las zonas y períodos señalados se permite la práctica de la escalada, el búlder y el rápel siempre que esté de acuerdo con el resto de normativa y con el permiso de la propiedad cuando el terreno sea privado.

4. RESOLUCIÓN DE 4 DE FEBRERO DE 2019, DE LA DIRECCIÓN GENERAL DE MEDIO AMBIENTE Y SOSTENIBILIDAD, POR LA QUE SE DETERMINAN LAS LÍNEAS ELÉCTRICAS AÉREAS DE ALTA TENSIÓN EXISTENTES EN ZONAS DE PROTECCIÓN APROBADAS EN LA COMUNIDAD DE MADRID, QUE NO SE AJUSTAN A LAS PRESCRIPCIONES TÉCNICAS ESTABLECIDAS EN EL REAL DECRETO 1432/2008, DE 29 DE AGOSTO, POR EL QUE SE ESTABLECEN MEDIDAS PARA LA PROTECCIÓN DE LA AVIFAUNA CONTRA 


\section{LA COLISIÓN Y LA ELECTROCUCIÓN EN LÍNEAS ELÉCTRICAS DE ALTA TENSIÓN}

El Real Decreto 1432/2008, de 29 de agosto, estableció diversas medidas para la protección de la avifauna contra la colisión y la electrocución en líneas eléctricas de alta tensión, estableciendo un marco normativo con normas de carácter técnico de aplicación a las líneas eléctricas aéreas de alta tensión con conductores desnudos ubicadas, en lo que denomina como "zonas de protección".

El artículo 4 del referido Real Decreto identifica dichas zonas de protección:

a) Los territorios designados como Zonas de Especial Protección para las Aves (ZEPA);

b) Los ámbitos de aplicación de los planes de recuperación y conservación elaborados por las comunidades autónomas para las especies de aves incluidas en el Catálogo Español de Especies Amenazadas o en los catálogos autonómicos;

c) Las áreas prioritarias de reproducción, alimentación, dispersión y concentración local de aquellas especies de aves incluidas en el Catálogo Español de Especies Amenazadas, o en los catálogos autonómicos.

Para delimitar dichas zonas, el apartado segundo de ese mismo artículo 4 establecía que el órgano competente de cada Comunidad Autónoma debía disponer la publicación en el correspondiente diario oficial de las zonas de protección existentes en su respectivo ámbito, fijando el plazo de un año para cumplir este cometido.

Pues bien, en la Comunidad de Madrid hubo de esperarse a la Resolución de 6 de julio de 2017 de la Dirección General del Medio Ambiente, que dispuso la delimitación y la publicación de las zonas de protección existentes en la Comunidad de Madrid.

De conformidad con este catálogo, la resolución de 4 de febrero de 2019 de la Dirección General de Medio Ambiente y Sostenibilidad recoge las líneas eléctricas de alta tensión existentes en las zonas de protección de la Comunidad de Madrid 
que no cumplen las medidas técnicas de protección y se identifican las que provocan mortalidad de avifauna y se dispone la comunicación de esta circunstancia a sus titulares.

A dichos efectos, el artículo 10 del Real Decreto 1432/2008, de 29 de agosto dispone la aplicación a quienes contravengan sus términos, el régimen sancionador de la Ley 54/1997, de 27 de noviembre, del Sector Eléctrico, entre cuyas infracciones muy graves se contempla:

"El incumplimiento, por parte del titular de las instalaciones, de su obligación de mantener las instalaciones en adecuadas condiciones de conservación e idoneidad técnica, siguiendo, en su caso, las instrucciones impartidas por la Administración competente y por el operador del sistema y gestor de la red de transporte o por los gestores de las redes de distribución, según corresponda, y los procedimientos de operación del sistema, cuando dicho incumplimiento ponga en riesgo la garantía de suministro o en peligro manifiesto a las personas, los bienes o al medio ambiente (artículo 60.16)"

En caso de que la lesión al medio ambiente no sea grave, el artículo 61.9 tipifica la misma conducta como infracción muy grave, que lleva aparejada una sanción de multa de hasta 6.000.000 de euros, mientras que en el caso de las muy graves puede ascender hasta los 30.000 .000 de euros.

\section{DECRETO POR EL QUE SE ADOPTAN EN LA CIUDAD DE MADRID LAS MEDIDAS DE RESTRICCIÓN DEL TRÁFICO Y DEL ESTACIONAMIENTO PREVISTAS EN EL PROTOCOLO DE ACTUACIÓN PARA EPISODIOS DE CONTAMINACIÓN POR DIÓXIDO DE NITRÓGENO EN LA CIUDAD DE MADRID}

A nivel local sin duda deben sin duda mencionarse las medidas adoptadas por el Ayuntamiento de Madrid contra la contaminación atmosférica. El viernes 4 de enero de 2019 (BOCM n³) publicó el Decreto 001, de 2 de enero de 2019, de la 
delegada del Área de Gobierno de Medio Ambiente y Movilidad, por el que se adoptan en la ciudad de Madrid las medidas de restricción del tráfico y del estacionamiento previstas en el protocolo de actuación para episodios de contaminación por dióxido de nitrógeno en la ciudad de Madrid, aprobado por Acuerdo de la Junta de Gobierno de 10 de diciembre de 2018.

Se aprobaba así el nuevo protocolo contra situaciones de elevada contaminación atmosférica en desarrollo de la Ley 34/2007, de 15 de noviembre, de Calidad del Aire y Protección de la Atmósfera, el Real Decreto 102/2011, relativo a la mejora de la calidad del aire.

Dicho protocolo ha venido acompañado de una elevada polémica, como consecuencia de la falta de entendimiento entre el Ayuntamiento de Madrid y el Gobierno de la Comunidad Autónoma quien, incluso, exigió su paralización tras una aprobación en falso en octubre de 2018 por no habérsele dado trámite previamente para informar su contenido. Por ello, el Ayuntamiento de la ciudad de Madrid se vio obligado a suspender su entrada en vigor para dar traslado de su contenido a la Comunidad Autónoma y que pudiera informarlo.

El Protocolo contempla tres niveles de actuación en función de las concentraciones de dióxido de nitrógeno que se registren o se prevean registrar:

- PREAVISO: cuando dos estaciones cualesquiera de una misma zona superan, simultáneamente, $180 \mu \mathrm{g} / \mathrm{m} 3$ durante dos horas consecutivas, o tres estaciones cualesquiera de la red de vigilancia superan, simultáneamente, $180 \mu \mathrm{g} / \mathrm{m} 3$ durante tres horas consecutivas.

- AVISO: cuando dos estaciones cualesquiera de una misma zona superan, simultáneamente, $200 \mu \mathrm{g} / \mathrm{m} 3$ durante dos horas consecutivas, o tres estaciones cualesquiera de la red de vigilancia superan, simultáneamente, $200 \mu \mathrm{g} / \mathrm{m} 3$ durante tres horas consecutivas.

- ALERTA: cuando tres estaciones cualesquiera de una misma zona (o dos si se trata de la zona 4 -situadas en los llamados pulmones verdes de Madrid, el Pardo y Casa de Campo) superan, simultáneamente, $400 \mu \mathrm{g} / \mathrm{m} 3$ durante tres horas consecutivas. 
A partir de estas situaciones, el Protocolo contempla 5 escenarios de actuación posibles con una serie de actuaciones para cada uno con intensidad creciente en lo que a los agentes contaminantes respecta:

ESCENARIO 1, que se produce con 1 día con superación del nivel de preaviso.

Este escenario contempla las siguientes actuaciones:

- Medidas informativas y de recomendación.

- Medidas de promoción del transporte público.

- Reducción de la velocidad a $70 \mathrm{~km} / \mathrm{h}$ en la M-30 y accesos.

ESCENARIO 2, se produce con 2 días consecutivos con superación del nivel de preaviso o 1 día con superación del nivel de aviso.

Se llevan a cabo las mismas actuaciones que el Escenario 1 y adicionalmente, entre otras, la prohibición de la circulación en el interior de la M-30 y por la M-30 a los vehículos a motor, incluidos ciclomotores, que no tengan la clasificación ambiental de "CERO EMISIONES", "ECO", "C" o "B" en el Registro de Vehículos de la Dirección General de Tráfico.

ESCENARIO 3: se produce con 3 días consecutivos con superación del nivel de preaviso o 2 días consecutivos con superación del nivel de aviso.

Las actuaciones programadas serían las mismas que el escenario 2, con el añadido de que se amplía a todo el término municipal la prohibición de la circulación a los vehículos a motor, incluidos ciclomotores, que no tengan la clasificación ambiental de "CERO EMISIONES", "ECO", "C" o "B" en el Registro de Vehículos de la Dirección General de Tráfico.

ESCENARIO 4. Este escenario se produce con 4 días consecutivos con superación del nivel de aviso, y motiva la realización de las actuaciones del escenario 3 y, además en lo relativo a la circulación se amplía a los vehículos con distintivo B. 
ESCENARIO ALERTA. Este escenario se activa con 1 día de nivel de alerta y conlleva la prohibición de circulación en todo el término municipal a los vehículos a motor, incluidos ciclomotores y taxis libres, que no tengan la clasificación ambiental de "CERO EMISIONES" o "ECO en el Registro de Vehículos de la Dirección General de Tráfico.

Una vez iniciado el episodio correspondiente, los distintos escenarios o su desactivación dependen de la evolución de los niveles de NO2 y las previsiones meteorológicas. Para prevenir a los ciudadanos, el Ayuntamiento de Madrid se ha comprometido a informar antes de las 12 horas del mediodía, salvo la aplicación del límite de velocidad del Escenario 1, que se aplicará a las 6 de la mañana, después de haberse producido la superación. Es decir, el primer día de activación del Protocolo, en el que no hay limitaciones de estacionamiento y circulación, se activa la noche anterior. Los siguientes escenarios se avisan antes de las 12 horas del día anterior. 